\title{
Comparative Docking Studies of Osthol Derivatives and Sorafenib on BRAF V599E Mutant Protein
}

\author{
S.Pavithra ${ }^{1}$, D. Velmurugan ${ }^{2}$, V.Srividhya ${ }^{1}$, P.Hemalatha ${ }^{1}$, V.P.Santhanakrishnan ${ }^{3, "}$ \\ ${ }^{1}$ Department of Plant molecular Biology and Bioinformatics, CPMB\&B, TNAU, Coimbatore-3 \\ ${ }^{2}$ Department of Crystallography and Biophysics University of Madras Guindy campus chennai-25 \\ ${ }^{3}$ Department of Plant Biotechnology, CPMB\&B, TNAU, Coimbatore-3 \\ *Corresponding Author: santhan_r@yahoo.co.uk
}

Copyright (C) 2014 Horizon Research Publishing All rights reserved.

\begin{abstract}
B-Raf, member of the RAF kinase family of serine/threonine-specific protein kinases. Mutations in the $B R A F$ gene may get inherited and cause birth defects resulting in cancer during the later life of humans. The mutation in BRAF includes V599E, V599D, L596V, L596R, G595R and F594L. Among this V599E involves about $92 \%$ of mutation. The inhibitors chosen for B-raf proto-oncogene serine/threonine-protein kinase mutant V599E were osthol and its derivatives, as, it is reported to be anticancerous compound. Among the ten compounds derived, docking result shows that Osthol Ritter product (ORP) possess more binding energy. To confirm this we have performed induced docking of ORP with the existing drug Sorafenib, both the compounds shows interaction with ASP 593 and GLU 500 and ORP's Glide score is similar to Sorafenib. There by we suggest that, the osthol ritter product as a drug candidate against cancer.
\end{abstract}

Keywords B-Raf, Osthol Ritter Product, Sorafenib, ADME

\section{Introduction}

Cancer, an unregulated growth of cells occurs mostly through mutations in the MAP kinase/ERKs signaling pathway. B-RAF is a protein that plays a major role in regulating the MAP kinase/ERKs signaling pathway. The mutations in B-Raf proteins such as, V599E, V599D, L596V, L596R, G595R and F594L are associated with cardiofaciocutaneous syndrome and with various cancers [1]. V599E is responsible for most of mutations that mimics phosphorylations, resulting in a high protein activity and Erk activation [2].

Sorafenib (Nexavar) is the existing drug for cancer which is approved by U.S. Food and Drug Administration (FDA), usually blocks the multiple kinases [3] and inhibits cell growth. Since most of these anti cancer drugs have side effects, it is proposed to use secondary metabolites and its derivatives as an alternate source against specific cancer cell lines.

Osthol (7-methoxy-8-(3-methyl-pent-2-enyl) coumarin) is a natural coumarin derivative isolated from Cnidii Monnieri [4,5] and Imperatoria ostruthium [6]. It is used as Chinese herbal medicine since ancient times [7]. The pharmacological study of the osthol includes anti-allergic [8], anti-osteoporotic [9], antifungal [10], anti-inflammatory [11] and anti-tumor [12] effects of osthol. The recent literatures reveals synthetic derivatives of osthol are used for hepatitis prevention [13] and for the anti breast cancer treatment [14]. In order to inhibit the mechanism of Braf mutations of V599E and to ensure the anti cancerous property of Osthol we performed in silico docking studies using osthol and nine of its derivatives [Table 1] against sorafenib.

\section{Material and Methods}

\subsection{Target Protein Structure Retrieval and Preparation}

The structures of mutant V599E B-Raf protein (PDB ID: 1uwj) X-RAY crystallographic structure at a resolution of $3.50 \AA$ was retrieved from Protein Data Bank (PDB) (http://www.rcsb.org/).

B-Raf structure was prepared using 'Protein Preparation wizard' in Schrodinger by removing the water molecules and adding hydrogen atoms. The structure was minimized and optimized using OPLS2005 force field. Energy minimization was done until the average root mean square deviation of non-hydrogen atoms reached $0.3[\AA]$.

\subsection{Selection of Ligand Molecules}

Osthol and Osthenol were retrieved from Pubchem [15] compound database (http://pubchem.ncbi.nlm.nih.gov/) and those structures were selected for the molecular docking studies. Along with the existing compounds, we have tried 
with other synthesized Osthol derivatives based on the literature search [16] and those were generated using chemsketch and validated by verifying its molecular formula and other properties. The existing cancer drug sorafenib was used for comparison with osthol derivatives.

\subsection{In Silico ADME and Lipinski Rule Prediction}

The ligand molecules were subjected to predict pharmacokinetic properties using the QikProp, an ADME prediction program from Schrödinger. This program provides all pharmogenetically relevant properties. Significant ADME properties such as octanol/water partition coefficient (QPlogPo/w), permeability through MDCK cells (QPlog MDCK), Qikprop predicted $\log \mathrm{IC}_{50}$ value for blockage of $\mathrm{K}+$ channels (QPlog HERG), QikProp predicted gut-blood barrier (QPPCaco) and human oral absorption percentage $(\mathrm{QP} \%)$ and skin permeability (QPlogKp) were predicted using the QikProp. Lipinski's rule of five (LROF) and rule of three was also verified, which is essential for rational drug designing.

\subsection{Ligand Preparation}

Osthol and its derivatives were prepared by using ligprep module in Schrodinger 2009. Ligprep produced different conformers of the ligand molecule based on ionization states, stereo chemistries and ring conformations. Ligands have been prepared using Epik for ionization and tautomerization. Partial atomic charges were computed by using the OPLS-2005 force field and it gave the corresponding low energy $3 \mathrm{D}$ conformers of the ligands.

\subsection{Docking Studies}

Docking of V599E - mutant B-RAF against osthol and its derivatives is done using Glide XP of Glide module in Schrodinger, Inc. The grid based ligand docking is performed based on the centroid of workspace ligand. Grid is generated with default parameters. Geometric or hydrogen bonding constraints were not introduced. Glide looks for constructive interactions between ligand molecule and receptor molecule and docking was carried over using the extra- precision (XP) algorithm. The best poses that glide generates passes through various filters and final pose of the ligand were refined to optimize the structure of ligand at the XP level of accuracy. The results of a Glide XP docking was visualized and analyzed in the Glide XP Visualizer panel. The final pose of ligand with good glide score was considered as best drug candidate and it is subjected to induced fit docking. The existing drug Sorafenib is also subjected to induced fit docking along with the candidate lead molecule.

\section{Results}

\subsection{Structure Determination}

The mutant V599E B-Raf protein induces cell division and acts as an oncogene and thereby causes cancer. This B-Raf protein complexed with BAY439006 is retrieved from the Protein databank (1UWJ) with 256 amino acid residues (Fig.1).

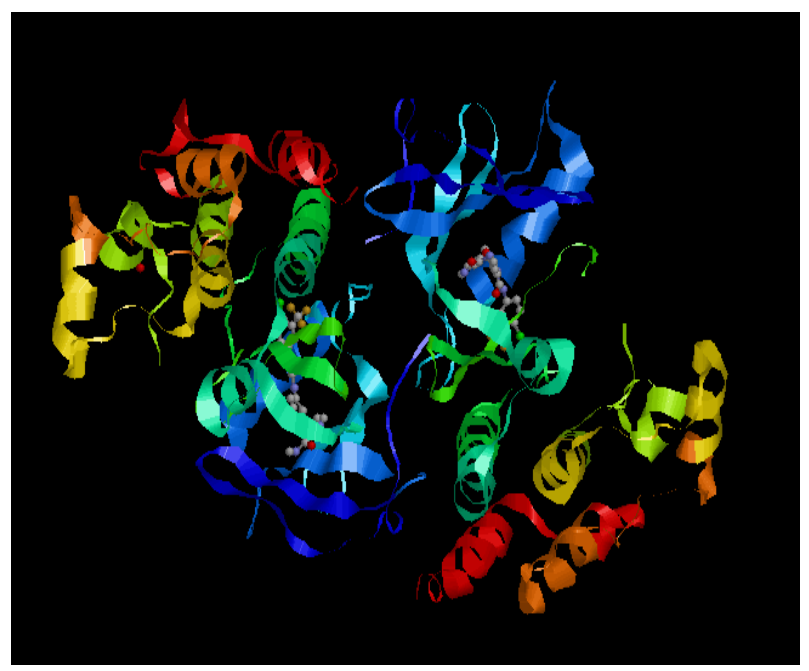

Figure 1. Structure of the complex mutant V599E -B- RAF

\subsection{Lead Molecule Generation}

Osthol and derivatives with the anti-cancerous property was chosen to inhibit the mutant B-Raf protein. The structure of the osthol and Osthenol was retrieved from pubchem. Other derivatives were generated using chemsketch and saved in mol file format. All the generated structure were validated by checking their IUPAC nomenclature. The structure and IUPAC name for the osthol and its derivatives were listed in the Table -1 .

\subsection{In Silico ADME Prediction and Biological Activity Prediction of Lead Molecules}

The Osthol and its derivatives were subjected to predict pharmacokinetic properties using the QikProp module from Schrodinger. The ADME prediction result shows that all the compounds have significant values suggesting its druggable characters. Lipinski rule of five suggests the drug likeliness of these compounds. Number of rotatable bonds and polar surface area illustrate the oral bioavailability of the molecules. The ADME properties and Lipinski rule of 5 were listed in the Table 2. 
Table 1. Osthol and its derivatives with IUPAC Nomenclature

\begin{tabular}{|c|c|c|}
\hline Name & Compounds & IUPAC NAME \\
\hline \multicolumn{3}{|c|}{ Osthol and Osthenol retrieved from Pubchem } \\
\hline $\begin{array}{c}\text { Osthol } \\
\text { CID } 10228\end{array}$ & & 7-methoxy-8-[3-methylpent-2-enyl]coumarin \\
\hline Osthenol & & \\
\hline CID: 5320318 & & 7-hydroxy-8-(3-methylbut-2-en-1-yl)-2H-chromen-2-one \\
\hline \multicolumn{3}{|c|}{ Osthol Derivatives taken from literature } \\
\hline $\begin{array}{l}\text { Osthol ethane thiol } \\
\text { complex (OET) }\end{array}$ & & 7-hydroxy-8-(3-methyl-3-thioethylbutyl)-coumarin \\
\hline Osthol cyclized derivative & & 7,8-(11,11-dimethyl-pyrano)coumarin \\
\hline $\begin{array}{l}\text { Osthol Ritter product } \\
\qquad(\mathrm{ORP})\end{array}$ & & 8-(3-methyl-3-benzoylamino-butyl)-7-methoxycoumarin \\
\hline $\begin{array}{l}\text { Osthol bromomethoxy } \\
\text { derivative (OBM) }\end{array}$ & & 7-methoxy-8-(3-methyl-3-methoxy-2-bromobutyl)coumarin \\
\hline $\begin{array}{l}\text { Osthol bromoacetoxy } \\
\text { derivative (OBA) }\end{array}$ & & 7-methoxy-8-(3-methyl-3-acetoxy-2-bromobutyl)coumarin \\
\hline $\begin{array}{c}\text { Osthol bromohydroxy } \\
\text { derivative (OBH) }\end{array}$ & & 7-methoxy-8-(3-methyl-3-hydroxy-2-bromobutyl) coumarin \\
\hline Epoxy osthol & & 7-methoxy-8-(3-methyl-2,3-epoxybutyl)coumarin \\
\hline
\end{tabular}




\begin{tabular}{|c|c|c|}
\hline $\begin{array}{c}\text { Osthol tandembromo } \\
\text { isomerized derivative } \\
\text { (OTBi) }\end{array}$ & 7-methoxy-8-(2-bromo-3-methyl-but-3-enyl)coumarin \\
\hline
\end{tabular}


Table 2. Lipinski rule and ADME properties of Ligand Molecules

\begin{tabular}{|c|c|c|c|c|c|c|c|c|c|c|c|c|}
\hline & Mol MW & Donorhb & $\begin{array}{c}\text { Accpt } \\
\text { HB }\end{array}$ & $\begin{array}{l}\text { Rule Of } \\
\text { Five }\end{array}$ & $\begin{array}{l}\text { Rule Of } \\
\text { Three }\end{array}$ & $\begin{array}{c}\text { Percent } \\
\text { Human Oral } \\
\text { Absorption }\end{array}$ & $\mathrm{QP} \log \mathrm{Po} / \mathrm{w}$ & $\begin{array}{l}\text { QPlog } \\
\text { MDCK }\end{array}$ & QPPCaco & QPlogKp & QPlogHERG & PSA \\
\hline Dihydro osthol & 246.305 & 0 & 3.25 & 0 & 0 & 100 & 3.149 & 1177.613 & 2230.838 & -1.834 & -4.646 & 46.123 \\
\hline Osthenol & 230.263 & 1 & 3.25 & 0 & 0 & 93.78 & 2.466 & 413.094 & 846.366 & -2.66 & -4.702 & 60.168 \\
\hline Osthol & 244.29 & 0 & 3.25 & 0 & 0 & 100 & 3.135 & 1131.839 & 2150.496 & -1.931 & -4.886 & 46.122 \\
\hline Osthol Bromo Acetoxy & 383.238 & 0 & 5.25 & 0 & 0 & 100 & 3.4 & 2123.226 & 2399.648 & -1.684 & -4.357 & 75.09 \\
\hline Osthol Bromo Hydroxy & 341.201 & 1 & 4 & 0 & 0 & 100 & 3.171 & 1137.902 & 1335.759 & -2.18 & -4.469 & 64.36 \\
\hline Osthol Bromo Isomerized & 323.186 & 0 & 3.25 & 0 & 0 & 100 & 3.616 & 2613.579 & 2618.008 & -1.596 & -4.572 & 45.311 \\
\hline Osthol Bromo Methoxy & 355.228 & 0 & 4 & 0 & 0 & 100 & 3.717 & 2321.4 & 2593.515 & -1.62 & -4.559 & 52.103 \\
\hline Osthol Cyclized & 230.263 & 0 & 3.25 & 0 & 0 & 100 & 2.484 & 1077.645 & 2055.058 & -2.227 & -4.36 & 46.878 \\
\hline Osthol Epoxy & 260.289 & 0 & 5.25 & 0 & 0 & 100 & 2.134 & 1193.332 & 2258.373 & -1.921 & -4.274 & 58.609 \\
\hline Osthol Ethane Thiol Complex & 292.392 & 1 & 3.75 & 0 & 0 & 100 & 3.394 & 1144.242 & 1687.294 & -1.819 & -4.221 & 59.383 \\
\hline Osthol Ritter Product & 365.428 & 1 & 5.75 & 0 & 0 & 100 & 4.222 & 1293.133 & 2432.582 & -0.981 & -5.597 & 76.552 \\
\hline Sorafenib & 464.831 & 3 & 6 & 0 & 1 & 95.591 & 4.097 & 1642.82 & 312.646 & -2.39 & -5.804 & 105.113 \\
\hline
\end{tabular}




\subsection{Molecular Docking}

The receptor V599E B-Raf protein complex protein is preprocessed, refined and optimized using the protein preparation module. The prepared structure posses the potential energy value $-530.383 \mathrm{kcal} / \mathrm{mol}$. The ligand molecules i.e., osthol and its derivatives were ionized and prepared at $\mathrm{pH} 7.0 \pm 2.0$ Epik state. The partial atomic charge is induced with OPLS-2005 force field. Then the grid is generated for the prepared protein molecule based on the workspace ligand molecule, with other default values. Docking of the ligand molecules against the receptor is performed using the extra- precision algorithm (Xp mode) in Schrodinger. [17]. The results of docking i.e., glide energy and dock score is given the Table 3 .

\subsection{Induced Fit Docking}

Among the 10 docked compounds as osthol Ritter product [18] showed good affinity towards the B-raf protein, and so it is subjected to induced fit docking and it was compared with the existing drug sorafenib. Induced fit docking studies was performed for Osthol Ritter Product and sorafenib with B-RAF proto-oncogene serine/threonine-protein kinase mutant V599E. 10 different poses were obtained in the results were given the table 4 . The results of best docked pose with its hydrogen bonding distance, glide energy and docking score are listed in the Table -4 . The docked structures were given in the figure 2 and 3 .

Table 3. Glide score, glide energy of extra precision docking studies were given below.

\begin{tabular}{|c|c|c|c|}
\hline S.NO & COMPOUND NAME & $\begin{array}{c}\text { GLIDE ENERGY } \\
(\mathrm{Kca} / \mathrm{Mol})\end{array}$ & DOCKING SCORE \\
\hline 1. & Osthol Ritter product (ORP) & -55.78 & -10.95 \\
\hline 2. & Osthol tandem bromo isomerized derivative(OTBi) & -34.53 & -9.86 \\
\hline 3. & Osthol ethane thiol complex(OET) & -40.45 & -9.83 \\
\hline 4. & Osthol bromoacetoxy derivative(OBA) & -35.76 & -9.76 \\
\hline 5. & Epoxy osthol & -35.67 & -9.60 \\
\hline 6. & Osthenol & -33.24 & -9.31 \\
\hline 7. & Osthol bromomethoxy derivative(OBM) & -33.65 & -8.89 \\
\hline 8. & Osthol bromohydroxy derivative(OBH) & -30.55 & -8.70 \\
\hline 9. & Osthol bromoacetoxy derivative & -30.07 & -8.85 \\
\hline 10. & Osthol cyclized derivative & -28.82 & \\
\hline
\end{tabular}

Table 4. Induced fit docking results of ORP and Sorafenib

\begin{tabular}{|c|c|c|c|c|c|}
\hline \multirow{2}{*}{ Pose } & Compound Name, Iupac Name & Hydrogen Bond & $\begin{array}{c}\text { Distance } \\
(\mathrm{a})\end{array}$ & $\begin{array}{c}\text { Glide } \\
\text { Energy } \\
(\mathrm{kcal} / \mathrm{mol})\end{array}$ & $\begin{array}{c}\text { Docking } \\
\text { Score }\end{array}$ \\
\hline \multirow{3}{*}{1} & Osthol Ritter product, & N-H-O(GLU500) & 3.37 & & \\
& 8-(3-methyl-3-benzoylamino-butyl)-7-methoxycoumarin & N-H-O(CYS531) & 3.18 & -68.78 & -11.29 \\
& & N-H-O(ASP593) & 2.99 & & \\
& & N-H-O(CYS531) & 2.78 & & \\
& & N-H-O(CYS531) & 3.10 & \\
& Sorafenib & N-H-O(GLU500) & 3.20 & -73.17 & -12.18 \\
& & N-H-O(GLU50) & 2.88 & & \\
\end{tabular}
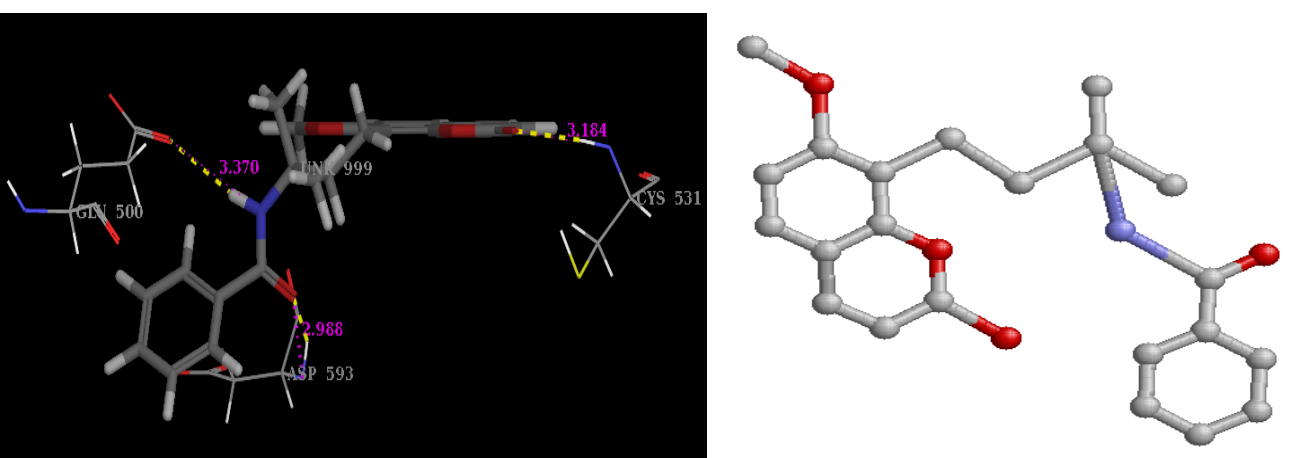

Figure 2. Hydrogen bond interactions of ORP with B-Raf mutant protein 

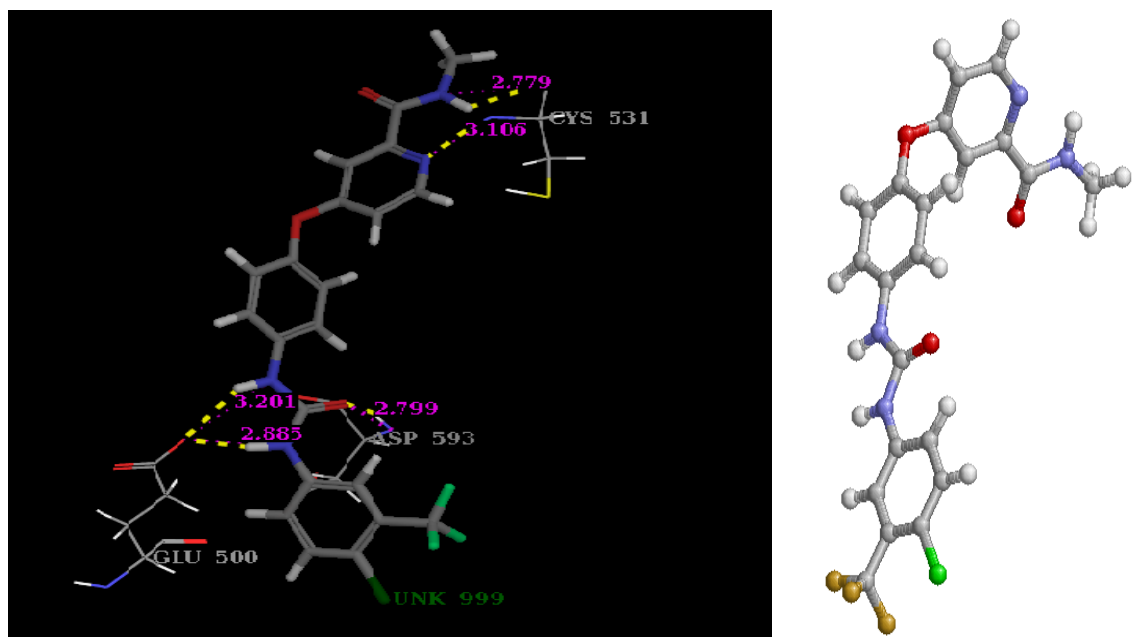

Figure 3. Hydrogen bond interactions of Sorafenib with B-Raf mutant protein

\section{Discussion}

The complex structure of B-RAF protein taken for analysis can be turned to its active conformation, while disrupting its interaction by clustering of most mutations to these regions. It is reported that the inhibition of this mutant protein will inhibit the cancer cell growth; hence an insilico work has been carried out to predict a compound that inhibits B-Raf protein. The active sites of the protein were found to be Glu500, Cys 531[12-13]. The Osthol and its derivatives were taken as a lead molecules. Good absorption is more likely and shows a druggable character as all the ligand molecule satisfies Lipinski's rule. Docking was performed for the ten compounds against B-Raf, it was found that Osthol Ritter product shows more affinity towards the B-raf protein, with lower glide energy and more interactions at active site. It posses the GLIDE ENERGY of -55.78 $\mathrm{Kcal} / \mathrm{Mol}$ and -10.95 dock score. Hence, ORP is subjected to induced fit docking and compared with the existing drug sorafenib. The first pose with glide energy -68.776971 and glide score - 11.289418 of ORP with B-Raf mutant protein and the second pose of sorafenib with glide energy -73.17 and glide score -12.18 were considered as the best. Both Osthol Ritter product and the drug Sorafenib had shown interaction with the identical residues GLU500, CYS531 and ASP593 as well as similar ADME properties proving the druggable property of ORP against B-Raf mutant protein.

\section{Conclusion}

The docking analysis of B-RAF proto-oncogene serine/threonine-protein kinase mutant V599E with osthol compounds and their derivatives showed potent result. Osthol Ritter product showed good binding affinity towards the target with lower glide energy and docking score. Osthol Ritter product had four interactions of which two residues ASP 593 and GLU 500 were the key residues and other two with hydrophobic residue CYS531 with glide energy -68.78 and glide score - 11.29. Both, Osthol Ritter product (ORP) and Sorafenib has shown the same hydrogen bonding interactions, suggesting the drug likeliness of ORP. Thus we suggest that, ORP could be used as a drug for the treatment of cancer after necessary clinical trials.

\section{Acknowledgements}

We would like to thank for the software facilities provided by the Centre of Advanced Study in Crystallography and Biophysics, University of Madras and Bioinformatics Centre, Department of Plant Molecular Biology and Bioinformatics, Centre for Plant Molecular Biology and Biotechnology, Tamil Nadu Agricultural University, Coimbatore-641 003.

\section{REFERENCES}

[1] Kathryn E. Mercer, Catrin A. Pritchard. Raf proteins and cancer: B-Raf is identified as a mutational target, Biochimica et Biophysica Acta, Vol.1653, 25- 40, 2003.

[2] Wan PT, Garnett MJ, Roe SM, Lee S, Niculescu-Duvaz D, Good VM, Jones CM, Marshall CJ, Springer CJ, Barford D, Marais R. Mechanism of Activation of the RAF-ERK Signaling Pathway by Oncogenic Mutations of B-RAF, Cell, Vol.116, 855-867, 2004.

[3] Liu L, Cao Y, Chen C, Zhang X, McNabola A, Wilkie D, Wilhelm S, Lynch M, Carter C. Sorafenib blocks the RAF/MEK/ERK pathway, inhibits tumor angiogenesis, and induces tumor cell apoptosis in hepatocellular carcinoma model PLC/PRF/5, Cancer Research , Vol. 66(24), 11851-11858, 2006.

[4] Shi Z, Wang F, Zhou W, Zhang P, Fan Y. Application of Osthol Induces a Resistance Response Against Powdery Mildew in Pumpkin Leaves, International Journal of Molecular Sciences, Vol.8, 1001-1012, 2007.

[5] Shi Z, Shen S, Zhou W, Wang F, Fan Y. Fusarium Graminearum Growth Inhibition Due to Glucose Starvation Caused by Osthol, International Journal of Molecular 
Sciences, Vol.9, 371-382, 2008.

[6] Raj K, Joshi PP, Bhakuni DS, Kapil RS, Popli SP. Synthesis of Auraptenol and Murrayone. Indian Journal of Chemistry, Vol.14(5), 332-335, 1976

[7] P.R. China. Various Pharmacopoeia of the People's Republic of China, Chemical Industry Press, Beijing, 62-63, 1997.

[8] Yamahara J, Kozuka M, Sawada T, Fujimura H, Nakano K, Tomimatsu T, Nohara T. Biologically active principles of crude drugs. Anti-allergic principles in "Cnidii monnieri", Chemical \& pharmaceutical bulletin, Vol.33, 1676-1680, 1985.

[9] Matsuda H, Tomohiro N, Ido Y, Kubo M. Anti-allergic effects of Cnidii Monnieri fructus (dried fruits of Cnidium monnierz) and its major component, osthol. Biological \& Pharmaceutical Bulletin, Vol.25, 809-812, 2002.

[10] Shi ZQ, Shen SG, Xu LL, Fan YJ. Inhibition Mechanism of Osthol to Plant Fungus Pathogens. Chinese Journal of Pesticide Science, Vol.6, 28-32, 2004..

[11] Liao JM, Zhu QA, Lu HJ, Li QN, Wu T, Huang LF. Effects of total coumarins of Cnidium monnieri on bone density and biomechanics of glucocorticoids induced osteoporosis in rats, Acta Phamacologica. Sinica, Vol. 8, 519-512, 1997.

[12] Szu-Yuan C, Chun-Sen H, Kun-Teng W, Min-Chieh W, Ching-Chiung W. Antitumor effects of Osthol from Cnidium monnieri: an in vitro and in vivo study, Phytotherapy Research,Vol.21, 226-230. 2006;
[13] Toshihiro O, Tadashi K, Shinic Y. Synthetic Derivatives of Osthole for the Prevention of Hepatitis, Journal of Medicinal Chemistry, Vol.3, 35-44, 2007

[14] Lisha You Rui An, Xinhong Wang, Yimin Li. Discovery of novel osthol derivatives as potential anti-breast cancer treatment, Bioorganic \& Medicinal Chemistry Letters, Vol. 20, 7426-7428, 2010.

[15] E.E. Bolton, Y. Wang, P.A. Thiessen, S.H. Bryant. PubChem: integrated platform of small molecules and biological activities, Annual Reports in Computational Chemistry, Vol.4, 217-241, 2008.

[16] Vichangal P. Santhanakrishnan, Raja Kalpana, Natarajan Sathishkumar, Srinivasan Narasimhan Chandrasekar Balakumaran. Molecular Docking Study on the Anti-inflammatory Effect of Osthol and Its Derivatives Towards Human p38alpha MAP kinase, International Journal of Bioinformatics, Vol. 4(1), 1-8, 2011

[17] RichardA. Friesner, Jay L. Banks, Robert B. Murphy, Thomas A. Halgren, Jasna J. Klicic, Daniel T. Mainz, Matthew P. Repasky, Eric H. Knoll, Mee Shelley, Jason K. Perry, David E. Shaw, Perry Francis, and Peter S. Shenkin. Surflex: Fully Automatic Flexible Molecular Docking Using a Molecular Similarity-Based Search Engine. Journal of Medicinal Chemistry, Vol.47, 1739-1749, 2004.

[18] Amirthasanjeevi L, Rajan S.S,Sridhar, B, Santhanakrishnan V.P., Narasimhan,S. Crystal Structure of 8-(3-Methyl-3-benzoylamino-butyl)-7-methoxycoumarin. Analytical Sciences 24: x29, 2008. 